\title{
Performance evaluation of distributed real-time scheduling systems using distributed production system simulator
}

\author{
Itsuo Hatono, Toshikazu Nishiyama, Motohide Umano and \\ Hiroyuki Tamura \\ Graduate School of Engineering Science, Osaka University \\ Toyonaka, Osaka 560, JAPAN \\ E-mail: hatono@sys.es.osaka-u.ac.jp
}

\begin{abstract}
This paper deals with a distributed simulation system to evaluate distributed realtime scheduling systems. To simulate various kinds of hybrid systems of distributed and centralized architectures, the distributed simulator in this paper consists of three kinds of schedulers: factory level scheduler, shop level scheduler, and equipment level scheduler. Moreover, the distributed real-time scheduling system is implemented as a virtual production system. Finally, we show the simulation results of the distributed real-time scheduling systems to evaluate the dispatching rules and the overhead for real-time scheduling.
\end{abstract}

\section{Keywords}

Distributed real-time scheduling, Virtual production system, Distributed simulation, Performance evaluation

\section{INTRODUCTION}

Flexible manufacturing has been developed to achieve multi-volume and least lot production. Flexible manufacturing is much more complex than the other production systems, because:

1. There exist alternative machine tools.

2. It is necessary to generate job transfer schedules, the schedules for operators of machine tools, and the schedules for tool supply, simultaneously.

3. There exist buffers at each machine tool and some temporal buffers.

In flexible manufacturing, in general, products are processed according to a job schedule obtained with off-line basis(Baker 1974). However, in flexible manufactur- 
ing, we often come across the failure of machine tools, a sudden change of production plans, and so on. In this case, real-time scheduling(Harmonosky and Robohn 1991) is more effective than off-line scheduling, because it is difficult to obtain a new schedule immediately in off-line scheduling. However, to develop a real-time scheduling system, we must take the architecture of the production management system into account, because in the real-time scheduling system, we must handle a huge amount of data in the production system in real-time(Parunak 1990). Some scheduling methods for such uncertain environments has been proposed (Blazewicz et al. 1996), but the methods are not taken the implementation on the real production systems into account.

Some architecture of next generation of production management systems are proposed (Okino 1992, Ueda 1992). Each production system are based on different concepts such as holonic(Okino 1992) and bionic(Ueda 1992), and so on, but almost all the production management system is developed as a distributed system. In such distribute production management systems, in general, there are no global management processes, because each manufacturing facility is considered as a subsystem that is independent of the other subsystems. Therefore, we need to allocate the real-time scheduling process on each subsystem without using a centralized scheduling process. In this case we need to develop cooperative scheduling protocols to negotiate between the scheduling processes.

In real-time scheduling, however, it is rather difficult to optimize the schedule and to estimate the performance of control computers and communication facilities theoretically, because the behavior of the production management systems are too complex. For each next generation of production management system, some real-time scheduling systems are proposed(Arai et al. 1996, Sugimura et al. 1996, Hatono et al. 1994), but the proposed real-time scheduling systems has not efficiently evaluated the performance.

In this paper, to develop the scheduling algorithms and protocols that are suitable to the production management systems, we evaluate the algorithms and protocols by computer simulations. However, it is difficult to develop the simulation system to verify and to evaluate the protocols by using conventional discrete events simulation technique, because it is too complex to develop the simulation model of the real-time scheduling system. To cope the difficulty, we developed a distributed simulation systems to evaluate a real-time scheduling system. The simulation system proposed in this paper can simulate the real-time scheduling system on not only a perfectly distributed production management systems but also hierarchical ones. Since we developed the simulation system as a virtual production system, we can simulate the detail behavior of the scheduling algorithms and protocols.

In this paper, first, we describe a brief introduction of distributed manufacturing systems and distributed real-time scheduling. Next, we describe a distributed simulation system to evaluate distributed real-time scheduling systems. Finally, we show the simulation results of the distributed real-time scheduling systems. 


\section{DISTRIBUTED MANUFACTURING SYSTEM}

In flexible manufacturing, since the production environment varies dynamically, the production management system must have functions to deal with failures of components, and reconfiguration or expansion of production facilities. To cope with these difficulties, it seems that it is more effective to construct a manufacturing system as a distributed system. In the centralized production system, the whole production system will stop, if the host CPU breaks down. However, in the distributed production system, the whole system will not stop, even if some components break down. In this paper, the production management system is assumed to be distributed to each component such as machining center, AGV and so on.

However, in many cases, the distributed production systems is not practical, because communication traffic between each scheduling process is too large if the number of machine tools in the production system is large. In those cases, the manufacturing systems are often constructed as hierarchical distributed systems. In this paper, we evaluate the performance of the real-time scheduling systems on not only the distributed production management systems but also the hierarchical ones.

\section{DISTRIBUTED REAL-TIME SCHEDULING SYSTEM}

To generate a schedule in real-time, it is necessary to resolve three kinds of conflicts as follows:

1. Selections of alternative machine tools (This is called routing of job),

2. Job selections for transporting,

3. Job selections from an input buffer.

In the distributed real-time scheduling system, these conflicts are resolved locally in the scheduling processes concerned with the conflicts. In this paper, we assume the following conditions:

1. In the distributed real-time scheduling system, we consider each machine tool as a subsystem. A scheduling process in each subsystem resolves the above conflicts locally.

2. Each scheduling process has only local information concerned with the machine tool managed by the scheduling process. By using communication facilities, the scheduling process can obtain the status of the other machine tools, and the whole system.

3. In flexible manufacturing, in general, it is difficult to manage the information concerned with each job, such as processing information, due dates, and so on, when the number of jobs to be processed becomes large. In this paper, the information is recorded in "tag," which is attached to each job. The information is read by each scheduling process and is used to resolve conflicts. 
In this paper, the information contained in tags consists of name of job, number of lot, processing information of jobs, history of processing, due date, sum of processing time up to the current time, processing start time, and degree of importance of due dates.

\section{DISTRIBUTED PRODUCTION SYSTEM SIMULATOR}

In this paper, the distributed production system simulator is designed based on the policies as follows:

1. We develop the each simulation program of the real-time scheduling on each machine tool as a process that is independent of the other processes. Therefore, it is not necessary to execute each simulation program on a computer.

2. Each simulation program can obtain the information for simulation of each machine tool and scheduling from the other simulation programs by inter-process communication.

3. We can simulate not only the distributed real-time simulation system but also the hierarchical ones.

To simulate various kinds of hybrid systems of distributed and centralized architectures, the distributed simulator in this paper consists of three kinds of schedulers: (1) factory level scheduler, (2) shop level scheduler, and (3) equipment level scheduler. In the followings, we describe the functions of each scheduler.

\subsection{Factory level scheduler}

We assumed that the factory level scheduler controls the whole production system. Therefore, there is only one factory level scheduler in the production system. In this paper, the factory level scheduler consists of the two kinds of schedulers as follows:

\section{Factory level job scheduler}

A factory level job scheduler controls the flows of jobs between the shops using the global information such as status of input and output buffers of each shop, utilization of each production equipment, and so on. When the factory level scheduler receives a scheduling requirement of a job from a shop level scheduler or a human manager of this factory, the factory level scheduler selects a shop for the next operation of the job, and returns the scheduling results the shop level scheduler or the manager for the scheduling requirement.

\section{Factory level AGV scheduler}

The factory level AGV scheduler controls the AGVs that convey jobs between the shops. The factory level AGV scheduler selects the alternative AGV to reduce the 


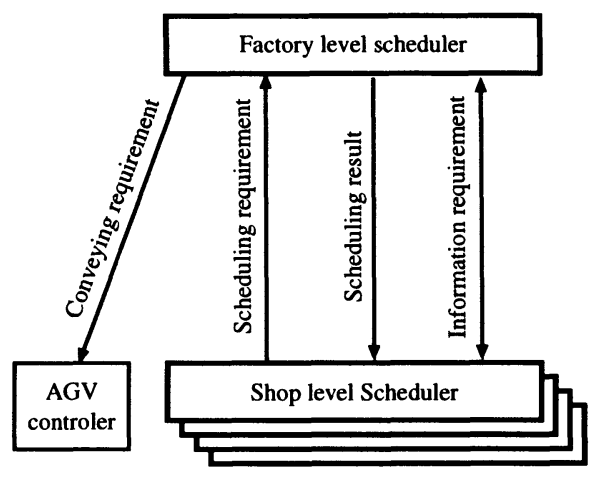

Figure 1 Overview of communications for scheduling in a factory level scheduler.

waiting times of jobs just after the selection of a shop for the next operation. Figure 1 shows the overview of communications for scheduling in a factory level scheduler.

\subsection{Shop level scheduler}

A shop level scheduler controls the flows of jobs in a shop, and controls the AGVs which convey jobs between the equipments based on the local information obtained in the shop. Furthermore, the shop level scheduler controls the flows of jobs among shops using contract net based cooperative scheduling algorithms(Hatono et al. 1994). In this case, it is not necessary to develop the factory level scheduler. The shop level scheduler consists of three scheduling modules as follows:

\section{Shop level cooperative scheduler}

A cooperative scheduler has scheduling functions as follows:

- The cooperative scheduler receives the processing requirement from the other scheduler such as the factory level scheduler and shop level schedulers, and send the scheduling requirement to the shop level job scheduler.

- The cooperative scheduler selects a shop for the next operation of jobs using the cooperative scheduling algorithm, if there exists no factory level scheduler.

- The cooperative scheduler generates the schedules for recovering from machine breakdowns and the emergency jobs.

\section{Shop level job scheduler}

A shop scheduler resolves the three kinds of conflicts in the shop: selections of alternative machine tool, job selections for conveying, and job selection from an input buffer of each machine tool in the shop. In this simulator, the appropriate dispatching rules are used to resolve the conflicts. 


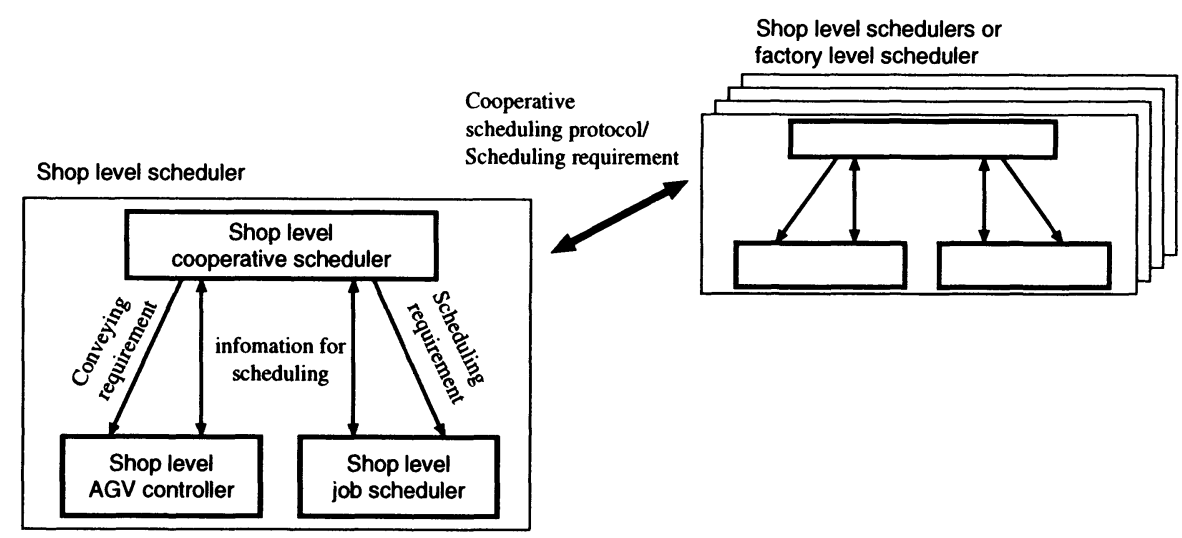

Figure 2 Overview of communications for scheduling in a shop level scheduler.

\section{Shop level AGV controller}

The shop level AGV controller controls the AGVs that convey jobs between the equipments. The shop level AGV scheduler selects the alternative AGV to reduce the waiting times of jobs just after the selection of the equipment for the next operation. Figure 2 shows the overview of communication for scheduling in a shop level scheduler.

\subsection{Equipment level scheduler}

A equipment level scheduler generates the processing orders of jobs in the input buffer. Furthermore, the equipment level scheduler requires the shop level scheduler to select the equipment of the next operation of each job.

We can develop the various kinds of hybrid systems of distributed and centralized architecture by omitting the appropriate level schedulers. Figure 3(a) (d) shows the examples of hybrid real-time scheduling systems.

\section{NUMERICAL EXAMPLES}

In this paper, we apply the distributed simulator to real-time scheduling systems which have the architecture shown in Figure 3 (a) $(d)$. Consider that the production system consists of 5 shops, each of which consists of 3 machine tools. In this production system, we assume that 10 kinds of jobs are processed and the number of processes of each job and the number of each job are 30 and 3, respectively. 
SS: Shop scheduler ES: Equipment scheduler

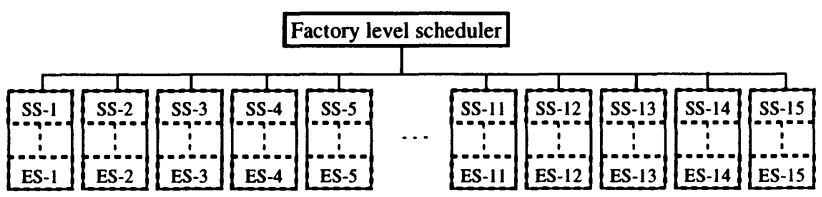

(a) Centralized real-time scheduling system.

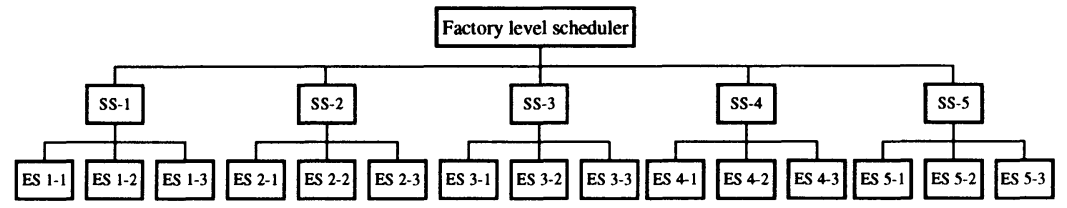

(b) Hierarchical Real-time scheduling system which consists of factory, shop, and equipment level schedulers.

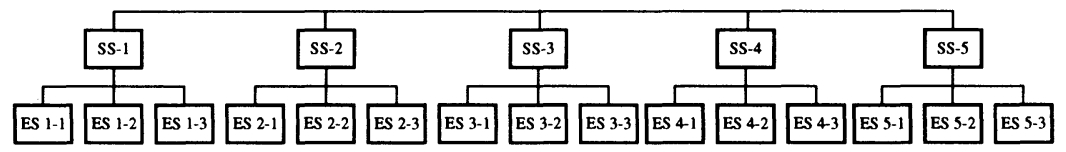

(c) Distributed real-time scheduling system which consists of shop and equipment level schedulers.

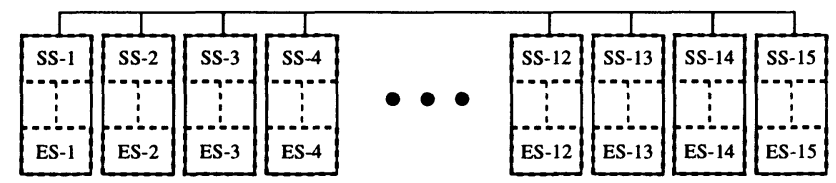

(d) Distributed real-time scheduling system which consists of shop and equipment level schedulers, which each shop level controller manages only one equipment level scheduler.

Figure 3 Examples of hybrid real-time scheduling system.

\subsection{Evaluation of scheduling methods}

In this numerical examples, we use the dispatching rules for selecting shop as follows:

- Dispatching rules for selecting an alternative shop:

Rule S-1: Select an alternative shop that contains the machine tool in which processing time of next operation of the job is shortest.

Rule S-2: Select an alternative shop that contains the machine tool in which the next operation of the job can be processed. 
Table 1 Dispatching rules and evaluation functions in numerical examples.

\begin{tabular}{ccccc}
\hline \multirow{2}{*}{$\begin{array}{c}\text { Real-time } \\
\text { scheduling } \\
\text { system }\end{array}$} & \multicolumn{3}{c}{$\begin{array}{c}\text { Dispatching rule } \\
\text { for selecting }\end{array}$} & $\begin{array}{c}\text { Evaluation } \\
\text { function for } \\
\text { contract net }\end{array}$ \\
\cline { 2 - 5 } & a shop & a machine tool & a job & - \\
\hline (a) & & M-1 M-3 & D-1 D-3 & - \\
\hline (b) & S-1, S-2 & M-1 M-3 & D-1 D-3 & - \\
\hline (c) & - & M-1 M-3 & D-1 DD-3 & F-2, F-3 \\
\hline (d) & - & - & D-1 D-3 & F-2, F-3 \\
\hline
\end{tabular}

- Dispatching rules for selecting an alternative machine tool:

Rule M-1: Select an alternative machine tool that has least number of jobs in input buffer.

Rule M-2: Select an alternative machine tool that contains the machine tool whose utilization is least.

Rule M-3: Select an alternative machine tool that contains the machine tool in which processing time of next operation of the job is shortest.

- Dispatching rules for selecting a job in input buffer:

Rule D-1: Shortest Processing Time (SPT)

Rule D-2: First Come First Serve (FCFS)

Rule D-3: Due Date (DDT)

- Evaluation functions for the contract net based cooperative scheduling protocols (Hatono et al. 1994):

Function F-1: Estimated shortest processing time of jobs

Function F-2: Number of jobs in a shop

Function F-3: Sum of processing times of jobs in machine tools that are available for the next processes of the jobs

Table 1 shows the rules and the evaluation functions that are used in the real-time scheduling systems shown in Figure 3 (a) (d)

Figure 4 (i) (iv) and Figure 5 (i) (iv) show the average of utilization of machine tools and the average of makespan of each job in the real-time scheduling systems shown in Figure 3 (a) $\sim(d)$, respectively. In these simulation results, the average utilization and waiting time in real-times scheduling system (a) are better than those in the other systems. This is because all the information for scheduling can be used in 


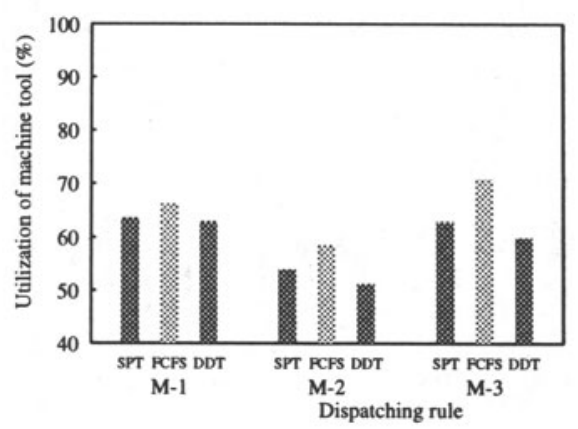

(i) Real-time scheduling system (a)

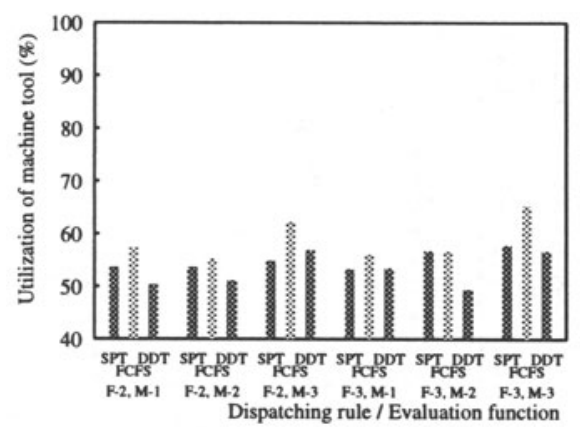

(iii) Real-time scheduling system (c)

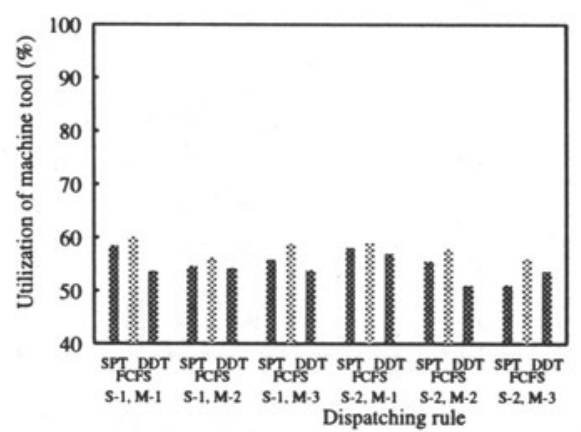

(ii) Real-time scheduling system (b)

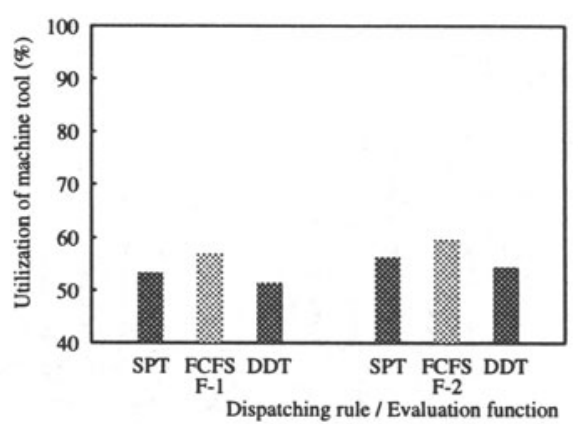

(vi) Real-time scheduling system (d)

Figure 4 Average utilization of machine tools

real-time scheduling system (a). However, the difference of the average utilization and makespan between real-time scheduling system (a) and (b) is relatively small. Therefore, we can guess that it is much more important to select appropriate dispatching rules in distributed real-time scheduling systems than that in centralized real-time scheduling systems.

Moreover, in the numerical experiments, DDT and SPT rules are effective for improvement of makespan of jobs, and FCFS rule is effective for improvement of utilization of machine tools. In distributed real-time scheduling system shown in Figure 3 (b) and (d), evaluation function F-2 is effective for improvement of both utilization of machine tools and makespan of jobs.

\subsection{Performance evaluation of communication facilities and computers}

Figure 6 shows the average number of operation on machine tool in every 20 time units when the number machine tools are varied. In Figure 6, real-time scheduling system (a), which is developed as a centralized system, the number of operation 


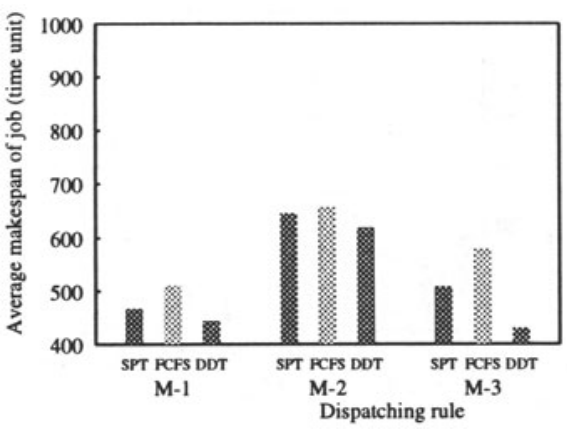

(i) Real-time scheduling system (a)

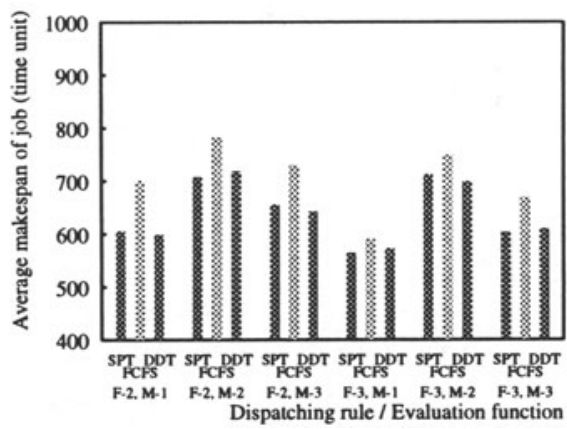

(iii) Real-time scheduling system (c)

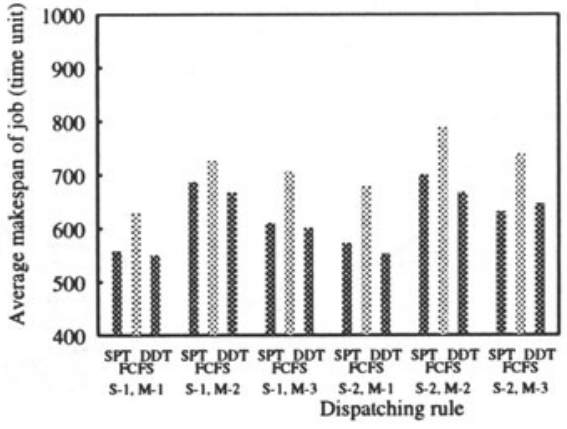

(ii) Real-time scheduling system (b)

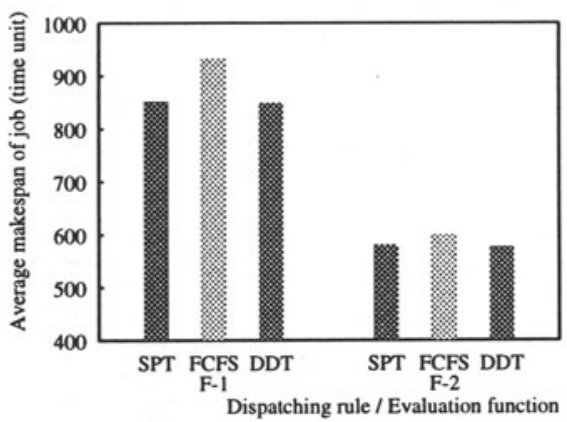

(vi) Real-time scheduling system (d)

Figure 5 Average makespan of jobs.

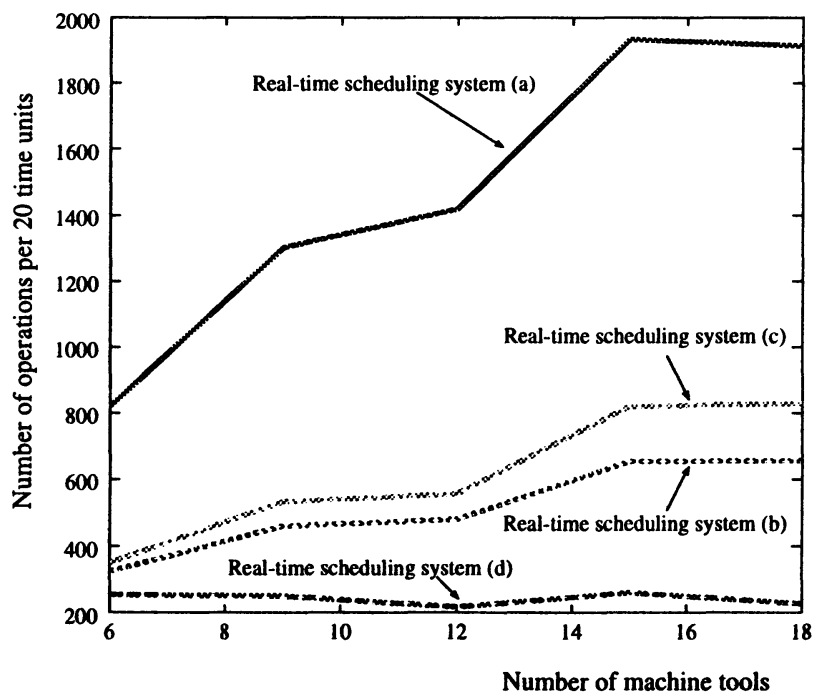

Figure 6 Average number of operations on machine tools in every 20 time units. 
increases in proportion to the increase of the number of machine tools. However, in real-time scheduling system (d), which is developed as a distributed system, the number of operation is not varied when the number of machine tools increase. Therefore, the overhead for scheduling in real-time scheduling system (d) is the least in the real-time scheduling system (a) (d). We can estimate that the performance of the computer of the factory level scheduler in real-time scheduling system (a) must be 10 times as much as that of real-time scheduling system (d).

\section{CONCLUSION}

In this paper, we develop a distributed simulation system to evaluate distributed real-time scheduling systems. The distributed real-time scheduling system is implemented as a virtual production system. Moreover, to simulate various kinds of hybrid systems of distributed and centralized architectures, the distributed simulator in this paper can simulate the real-time scheduling system which consists of three kinds of schedulers: factory level scheduler, shop level scheduler, and equipment level scheduler.

Finally, we show the simulation results of the distributed real-time scheduling systems to evaluate the dispatching rules for scheduling and the overhead for scheduling in each scheduler. Further research might be focused on the efficiency of the simulator by applying a larger scale production systems and development of the graphical user interface of the simulator.

\section{REFERENCES}

Arai, E., L. Jihong and S. Amnuay (1996). Distributed production system to realize flexible scheduling. In: Proceedings of Japan/USA Symposium on Flexible Automation. Boston. pp. 1365-1372.

Baker, K.R. (1974). Introduction to Sequencing and Scheduling. John Wiley and Sons. New York.

Blazewicz, J., K.H. Ecker, E. Pesch, G. Schmidt and J. Weglarz (1996). Scheduling Computer and Manufacturing Processes. Springer-Verlarg.

Harmonosky, C.M. and S.F. Robohn (1991). Real-time scheduling in computer integrated manufacturing: Review of recent research. International Journal of Computer Integrated Manufacturing 4(6), pp. 331-340.

Hatono, I., K. Tachibana, M. Umano and H. Tamura (1994). Distributed real-time scheduling for flexible manufacturing. In: Proceedings of JAPAN-U.S.A. Symposium on Flexible Automation - A Pacific Rim Conference -. Kobe. pp. 803-810.

Okino, N. (1992). A prototyping of bionic manufacturing system. In: Proceedings of the ICOOMS '92. pp. 297-302.

Parunak, H.V.D. (1990). Distributed AI and manufacturing control: Some isuues and insights. In: Decentralized A.I. (Y. Demazeau et al., Eds.). pp. 81-101. NorthHolland. Amsterdam. 
Sugimura, N., M. Hiroi, T. Moriwaki and K. Hozumi (1996). A study on holonic scheduling for manufacturing system of composite parts. In: Proceedings of Japan/USA Symposium on Flexible Automation. Boston. pp. 1407-1410.

Ueda, K. (1992). An approach to bionic manufacturing systems based on DNC-Type information. In: Proceedings of the ICOOMS '92. pp. 303-308.

\section{BIOGRAPHY}

Itsuo Hatono received the B.S. and M.S. degrees in engineering from Osaka University, Osaka, Japan, in 1984 and 1986, respectively, and the Ph.D. degree in engineering from Osaka University in 1994. Currently, he is an assistant professor of Department of Systems and Human Science, Graduate School of Engineering Sciences, Osaka University. His research interests include production scheduling for complex and large production systems, and modeling of discrete event systems such as manufacturing systems and computer networks.

Toshikazu Nishiyama received B.S. and M.S. degrees in engineering from Osaka University, Osaka, Japan, in 1994 and 1996, respectively. Currently, he is a research Engineer of Matsushita Electric Industrial Co., Osaka Japan. His research interests in Osaka University included the development of real-time scheduling systems and distributed production system simulators.

Motohide Umano received the B.S. degree in 1974, the M.S. degree in 1976 and the Dr. of Engineering degree in 1979 in Information and Computer Sciences from Osaka University, Japan. Currently, he is a professor of Department of Mathematics and Information Sciences, College of Integrated Art and Sciences, Osaka Prefecture University. His current research interests include fuzzy-set oriented programming languages, fuzzy databases, fuzzy expert systems and fuzzy knowledge information processing.

Hiroyuki Tamura received the B.S. and M.S. degrees in engineering from Osaka University, Osaka, Japan, in 1962 and 1964, respectively, the M.S. degree in EngineeringEconomic Systems from Stanford University, Stanford, California, U.S.A., in 1968, and the Ph.D. degree in engineering from Osaka University in 1971. Currently, he is a professor of Department of Systems and Human Science, Graduate School of Engineering Science, Osaka University. His research interests center on the systems methodology for large-scale systems such as modeling, control and decision making, and its applications to manufacturing systems and public systems. 